\title{
STOMACH
}

\section{Bile reflux gastritis and intestinal metaplasia at the cardia}

\author{
M F Dixon, N P Mapstone, P M Neville, P Moayyedi, A T R Axon
}

Gut 2002;51:351-355

See end of article for authors' affiliations

.......................

Correspondence to:

Professor M F Dixon,

Department of

Histopathology, General

Infirmary at Leeds, Leeds

LS 1 3EX, UK:

miked@pathology.leeds.ac.uk

Accepted for publication

28 January 2002
Background and aims: Intestinal metaplasia (IM) at the cardia is likely to be a precursor of cardia cancer. Previous work has shown that it is associated with chronic inflammation attributable to either gastro-oesophageal reflux disease (GORD) or Helicobacter pylori infection. An alternative aetiological factor is bile reflux. Duodenogastric reflux brings about histological changes in the gastric mucosa that can be graded and used to calculate a bile reflux index (BRI). We used the BRI to assess whether reflux of bile plays a part in the development of cardia IM.

Methods: Histological changes in simultaneous gastric antrum and cardia biopsies from 267 dyspeptic patients were independently graded by two pathologists. The association between cardia IM and age, sex, clinical group, $H$ pylori status, increased BRI (>14), and inflammation at the cardia were evaluated using logistic regression.

Results: A total of 226 patients had adequate cardia and antral biopsies; 149 had GORD and 77 had non-ulcer dyspepsia. Cardia IM was present in 66 (29\%) patients, of whom 28 (42\%) had complete IM. Increasing age, male sex, chronic inflammation, and a high BRI emerged as significant independent associations with cardia IM. Clinical group and $H$ pylori status were not independent risk factors. Conclusions: Histological evidence of bile reflux into the stomach is associated with cardia IM. This could have an important bearing on carcinogenesis at this site.
$\mathrm{T}$ he cardia is an area of controversy. Conventionally this "small and ill defined" region is described as a zone $0.5-3$ cm long "just distal to the lower end of the oesophagus" where it is interposed between oxyntic mucosa and squamous epithelium. ${ }^{12}$ However, its existence as a normal anatomical structure has been challenged. Chandrasoma and colleagues ${ }^{3}$ claim that the cardia is absent in truly normal individuals so that oxyntic mucosa abuts directly onto squamous epithelium. They argue that cardia-type mucosa is always acquired and is a consequence of gastro-oesophageal reflux disease (GORD). Cardia mucosa closely resembles the gastric antrum and it is suggested that the "acquired" cardia is an example of gastric metaplasia in response to acid reflux. ${ }^{3}$

Intestinal metaplasia (IM) at the cardia has attracted further controversy. Its very frequency (15-22\% of dyspeptic subjects with an endoscopically normal lower oesophagus ${ }^{4-6}$ ) argues against it being closely associated with cardia cancer yet by analogy with the stomach, cardia IM is considered to be a premalignant condition. ${ }^{7}$ While gastric metaplasia can be readily understood as an adaptive response of squamous epithelium to acid injury, it seems likely that intestinal metaplasia is triggered by other factors. Previous work has implicated gastric Helicobacter pylori infection and the associated "carditis" ${ }^{8}$ but other investigators have dismissed this in favour of GORD. ${ }^{9}$ The latter conclusion does not necessarily implicate acid as the sole causative agent. There is increasing evidence that GORD is accompanied by duodenogastric reflux so that the oesophageal refluxate can contain bile and other components of pancreaticoduodenal secretion. ${ }^{10}$ It would be much more plausible for the change from gastric to intestinal phenotype at the cardia to be related to bile than to acid reflux alone.

To investigate the role of bile reflux in the causation of cardia IM, we used a histological indicator of bile injury to the antrum - the bile reflux index (BRI) ${ }^{11}$-along with the extent of cardia inflammation and H pylori status to search for possible aetiological associations. Additionally, as the various subtypes of IM at the cardia are likely to carry different risks for progression to cancer, we sought to relate these to the same factors.

\section{PATIENTS AND METHODS}

Over a two year period, the Department of Pathology at Leeds General Infirmary received simultaneous biopsies of the gastro-oesophageal junction and gastric mucosa from 267 patients. The majority (192 cases) consisted of patients referred directly by their general practitioner to an open access dyspepsia clinic. Suitable patients were seen as part of an assessment procedure prior to possible recruitment into a study evaluating the symptomatic response of $H$ pylori negative dyspepsia to pantoprazole. Patients aged $18-80$ years were considered. Those taking non-steroidal antiinflammatory drugs or proton pump inhibitors less than four weeks prior to endoscopy, those who had undergone prior gastric or oesophageal surgery, and patients who were known to have been $H$ pylori positive in the past or had received $H$ pylori eradication therapy were excluded. Prior to endoscopy, patients were assessed by an experienced gastroenterologist $(\mathrm{PMN})$ and categorised on clinical grounds as having either non-ulcer dyspepsia (NUD) or GORD. The diagnosis of NUD was based on a clinical presentation of predominant epigastric pain or abdominal symptomatology (for example, bloating or nausea) with clinically insignificant or absent heartburn. The diagnosis of GORD was based on a clinical presentation of significant heartburn or acid reflux with or without epigastric pain. At endoscopy, four quadratic biopsies were taken from immediately below the squamocolumnar junction ( $\mathrm{Z}$ line), two biopsies from the gastric body (one on the lesser curve and one on the greater curve), one from the incisura angularis, and two from the prepyloric antrum. The local ethics committee gave approval for the biopsies with prior consent.

Classification of patients into NUD and GORD was modified after endoscopy. Patients with active ulcer disease, macroscopic evidence of Barrett's oesophagus, severe (>grade 3 ) oesophagitis, and neoplasia were excluded. Those patients

Abbreviations: IM, intestinal metaplasia; GORD, gastro-oesophageal reflux disease; NUD, non-ulcer dyspepsia; $B R I$, bile reflux index; $A B / P A S$, alcian blue/periodic acid-Schiff; 

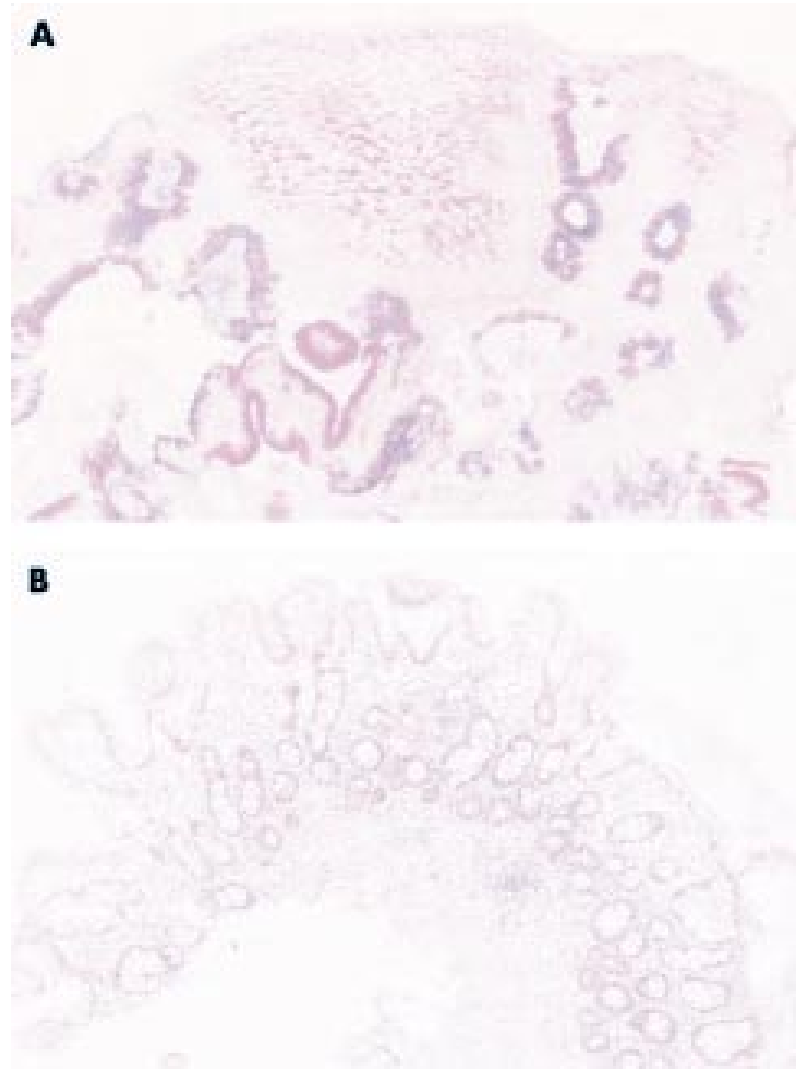

Figure 1 (A) Cardia biopsy (squamocolumnar junction) stained with alcian blue/periodic acid-Schiff for mucins showing widespread incomplete-type intestinal metaplasia. (B) Antral biopsy from the same patient (haematoxylin-eosin) showing widespread intestinal metaplasia (complete type) (grade=3), mild lamina propria oedema (grade $=1$ ), and mild chronic inflammation (grade $=1$ ). Helicobacters were not present in this case. Based on these findings the bile reflux index was 20 .

who had been classified as NUD on clinical grounds who on endoscopy were found to have Savary grade 2-3 oesophagitis were reclassified as GORD on the grounds that significant endoscopic oesophagitis is a more powerful predictor of GORD than clinical assessment.

The remaining 75 patients had cardia biopsies taken for some clinical indication, for example as an adjunct to the his- tological diagnosis of GORD or because an endoscopic abnormality (inflammation or nodularity) was seen at the junction. These patients also had no evidence of Barrett's oesophagus, active peptic ulceration, or previous gastric surgery, and at endoscopy at least two biopsies from immediately below the $\mathrm{Z}$ line, two corpus, and two antral biopsies were taken.

These 75 patients were not assessed prospectively but their notes were obtained and scrutinised retrospectively (PMN). Clinical histories had been taken at the time of endoscopy and were documented in the notes with the endoscopy report. These patients were categorised into GORD and NUD groups using the same criteria as for the prospectively recruited patients.

\section{Histological assessment}

Biopsies were fixed in 10\% formalin, processed, sectioned at three levels, and stained by haematoxylin-eosin. Additional sections taken at the second level from all biopsies were stained with alcian blue/periodic acid-Schiff (AB/PAS) to demonstrate IM and by the modified Giemsa stain for $H$ pylori. The cardia and gastric biopsies were assessed separately by two pathologists (NPM and MFD, respectively) who were "blind" to each other's findings and to the clinical details. The "cardia" biopsies were categorised as either squamous, oxyntic, oxyntocardiac transitional zone, or cardia type. We accepted as cardia those biopsies composed of gastric-type foveolar and surface epithelium overlying either antral-like mucous glands devoid of parietal cells or mucous glands with occasional parietal cells. The transitional mucosa was characterised by an admixture of mucous and oxyntic glands. A case was only included when there was at least one cardia biopsy and at least one antral biopsy adequate for assessment.

The presence of acute inflammation (that is, neutrophil polymorph infiltration), chronic inflammation (CI) lymphocytes and plasma cells-and IM in the cardia and antrum were graded on a 0-3 scale (absent, mild, moderate, and marked), while additionally in the antrum, lamina propria oedema (Oed) and $H$ pylori colonisation density $(H p)$ were graded. The grading approach was analogous to that described in the updated Sydney system ${ }^{12}$ although small numbers of mononuclear cells at the cardia were considered "normal" (see below). The Giemsa stained corpus biopsies were also examined before a case was declared $H$ pylori negative. IM was defined by the finding of fully formed goblet cells in the AB/PAS stain. When detected, a new section was cut and stained with Gomori's aldehyde fuchsin technique to distinguish between type II and III (sulphomucin positive) IM. ${ }^{13}$

Table 1 Grades of acute and chronic cardia inflammation according to clinical group, Helicobacter pylori (Hp) status, and bile reflux index (BRI). Grades 1, 2, and 3 are equivalent to a mild, moderate, and marked increase. For acute inflammation grade $\mathrm{O}=$ absence of polymorphs; for chronic inflammation $\mathrm{O}=$ "normal" numbers of lymphocytes and plasma cells (see text)

\begin{tabular}{|c|c|c|c|c|c|c|c|c|c|}
\hline \multirow[b]{2}{*}{ Group } & \multirow[b]{2}{*}{$\mathrm{n}$} & \multicolumn{4}{|c|}{ Acute } & \multicolumn{4}{|c|}{ Chronic } \\
\hline & & 0 & 1 & 2 & 3 & 0 & 1 & 2 & 3 \\
\hline NUD & 77 & 59 & 14 & 4 & 0 & 35 & 26 & 14 & 2 \\
\hline GORD & 149 & 122 & 20 & 7 & 0 & 83 & 44 & 20 & 0 \\
\hline $\mathrm{Hp}+$ & 44 & 10 & 24 & 10 & 0 & 2 & 15 & 25 & 2 \\
\hline $\mathrm{Hp}-$ & 182 & 171 & 10 & 1 & 0 & 118 & 55 & 9 & 0 \\
\hline$B R I \leqslant 14$ & 188 & 151 & 29 & 8 & 0 & 105 & 57 & 25 & 1 \\
\hline$B R I>14$ & 38 & 30 & 5 & 3 & 0 & 16 & 13 & 9 & 1 \\
\hline
\end{tabular}

GORD, gastro-oesophageal reflux disease; NUD, non-ulcer dyspepsia.

Acute and chronic inflammation of grades 2 and 3 were combined for all analyses.

NUD versus GORD: acute, Pearson $\chi^{2}=0.97, \mathrm{df}=2, \mathrm{p}=0.62$; chronic, Pearson $\chi^{2}=3.08, \mathrm{df}=2, \mathrm{p}=0.21$.

$H p+$ versus $H p-$ : acute, Pearson $\chi^{2}=114.92, d f=2, p<0.0001$; chronic, Pearson $\chi^{2}=94.95, d f=2$

$\mathrm{p}<0.0001$.

$B R I \leqslant 14$ versus $B R I>14$ : acute, Pearson $\chi^{2}=0.98, d f=2, p=0.61$; chronic, Pearson $\chi^{2}=4.72, d f=2$, $\mathrm{p}=0.09$. 
Table 2 Association between intestinal metaplasia (IM) at the cardia and sex, increased bile reflux, Helicobacter pylori status, clinical group, and presence of acute and chronic inflammation at the cardia

\begin{tabular}{|c|c|c|c|c|c|c|c|}
\hline Variable & $\begin{array}{l}\text { Total } \\
\text { No }\end{array}$ & $\begin{array}{l}\% \text { IM at } \\
\text { cardia }\end{array}$ & $\begin{array}{l}\text { Unadjusted } \\
\text { odds ratio }\end{array}$ & $95 \% \mathrm{Cl}$ & $\begin{array}{l}\text { Adjusted } \\
\text { odds ratio* }\end{array}$ & $95 \% \mathrm{Cl}$ & $p$ Value \\
\hline \multicolumn{8}{|l|}{ Sex } \\
\hline Female & 130 & 24.6 & 1.00 & & 1.00 & & \\
\hline Male & 96 & 35.4 & 1.68 & $0.94-2.99$ & 2.67 & $1.36-5.27$ & 0.004 \\
\hline \multicolumn{8}{|l|}{ BRI } \\
\hline$\leqslant 14$ & 188 & 23.9 & 1.00 & & 1.00 & & \\
\hline$>14$ & 38 & 55.3 & 3.92 & $1.91-8.08$ & 2.62 & $1.17-5.86$ & 0.019 \\
\hline \multicolumn{8}{|l|}{ H pylori } \\
\hline Negative & 182 & 25.8 & 1.00 & & 1.00 & & \\
\hline Positive & 44 & 43.2 & 2.18 & $1.10-4.32$ & 1.03 & $0.45-2.36$ & 0.95 \\
\hline \multicolumn{8}{|l|}{ Group } \\
\hline GORD & 149 & 28.2 & 1.00 & & 1.00 & & \\
\hline NUD & 77 & 31.2 & 1.15 & $0.63-2.10$ & 1.06 & $0.53-2.09$ & 0.87 \\
\hline \multicolumn{8}{|l|}{ Cardia $\mathrm{Cl}$} \\
\hline No & 119 & 18.5 & 1.00 & & 1.00 & & \\
\hline Yes & 107 & 41.1 & 3.08 & $1.69-5.62$ & 2.87 & $1.37-6.02$ & 0.005 \\
\hline Cardia Al & & & & & N/A & N/A & N/A \\
\hline No & 181 & 24.3 & 1.00 & & & & \\
\hline Yes & 45 & 48.9 & 2.98 & $1.52-5.86$ & & & \\
\hline \multicolumn{8}{|c|}{$\begin{array}{l}\text { *Factors mutually adjusted for each other and age in a logistic regression model. Age was statistically } \\
\text { significant in the model (adjusted odds ratio=1.05/year ( } 95 \% \text { confidence interval (CI) } 1.02-1.07) \text {; } \\
p=0.0007) \text {. } \\
\text { N/A, not applicable as acute and chronic inflammation at the cardia exhibited colinearity. Including chroni } \\
\text { inflammation at the cardia resulted in the model with the most explanatory power. } \\
\text { GORD, gastro-oesophageal reflux disease; NUD, non-ulcer dyspepsia; Cl, chronic inflammation; Al, acute } \\
\text { inflammation. }\end{array}$} \\
\hline
\end{tabular}

Histological findings in the antrum were used to calculate the BRI. BRI was originally derived by stepwise logistic regression analysis of the histological grades found in antral biopsies from 350 subjects in whom gastric juice bile acid levels had been measured. Following analysis, an index comprising $(7 \times \mathrm{Oed})+(3 \times \mathrm{IM})+(4 \times \mathrm{CI})-(6 \times \mathrm{Hp})$ gave the best prediction of a raised gastric juice bile acid concentration. An index above 14 had a sensitivity of $70 \%$ and a specificity of $85 \%$ for a bile acid level $>1.00 \mathrm{mmol} / \mathrm{l}$ (the upper limit of "physiological" $^{\prime \prime}$ reflux). ${ }^{11}$

\section{Statistical analysis}

The effect of clinical group (GORD or NUD), H pylori status, and increased bile reflux on acute and chronic inflammation at the cardia was assessed using the Pearson $\chi^{2}$ test. The association between IM at the cardia and sex, $H$ pylori status, increased bile reflux, clinical group, and the presence of acute and chronic inflammation at the cardia were evaluated using Fisher's exact test. The association between these factors and type of IM was assessed using Fisher's exact test while the association between age and IM was assessed using the Student's independent $t$ test. Independent risk factors for IM at the cardia were evaluated using logistic regression with cardia IM as the independent variable. The proportion of the variance explained by the logistic regression model was calculated using Nagelkerke $r^{2}$. A p value $<0.05$ was considered statistically significant for all analyses, and calculations were performed using SPSS version 9.0 (SPSS Inc. Chicago, Illinois, USA).

\section{RESULTS}

In 39 of 267 patients biopsied, no cardia-type mucosa was identified, and in a further two cases the antral biopsies were inadequate or missing. Thus there were 226 patients with cardia mucosa available (range 1-4 biopsies, median 2, mean 1.98 ) and at least one adequate biopsy of antral mucosa (see fig 1). Of these, 149 patients were considered to have GORD and 77 had NUD. The gastric biopsy findings from 47 of these NUD patients who were $H$ pylori negative were used in the control group of a previous study on bile reflux gastritis. ${ }^{14}$ With regard to $H$ pylori status, 182 of 226 patients were negative and $44(19.5 \%)$ were positive. There were no cases where the corpus biopsies were positive and the antral biopsies were negative for helicobacters. Concerning the BRI, 188/226 patients had a BRI $\leqslant 14$ while 38 had a BRI $>14$. While inflammation at the cardia showed no significant differences between subjects with GORD or NUD, or with an elevated BRI, H pylori positive cases were highly significantly different from negative patients for both acute and chronic inflammation (table 1).

IM was found at the cardia in 66 patients $(29 \%)$; in 28 the cardia IM was predominantly (if not exclusively) type I, in 25 it was type II, and in 13 it was type III.

Unadjusted analyses revealed that $H$ pylori infection, BRI $>14$, and acute and chronic inflammation at the cardia were associated with IM (table 2 ). There was a trend for male sex to be associated with cardia IM but this did not reach statistical significance, while there was no difference between GORD and NUD patients (table 2). Patients with IM at the cardia were older (median age 57 years) than patients without IM (median 47 years) and the difference in mean age was 9.7 years (95\% confidence interval (CI) 5.6-13.7). H pylori infection and BRI >14 were also associated with increasing age and it is possible that these factors are acting as markers for older patients. To evaluate independent risk factors for IM at the cardia, age, sex, clinical group, BRI $\leqslant 14$ or $>14, H$ pylori status, and chronic inflammation at the cardia were entered into a logistic regression model. Acute inflammation was omitted from the model as this exhibited strong colinearity with chronic inflammation. Age, sex, BRI >14, and chronic inflammation remained independent risk factors for cardia IM but $H$ pylori status became non-significant (table 2 ). The model explained $25.5 \%$ of the variance of the data. $H$ pylori status was strongly associated with chronic inflammation and the lack of a statistically significant relationship between $H$ pylori infection and cardia IM could be due to colinearity. This is not the case however as $H$ pylori was not associated with cardia IM in a logistic model that only included age (odds ratio $1.7 ; 95 \%$ CI $0.8-3.4 ; \mathrm{p}=0.17$ ).

The influence of $H$ pylori status, BRI > 14, sex, clinical group, and chronic and acute inflammation on the type of cardia IM 
Table 3 Association between incomplete intestinal metaplasia (types II and III IM), sex, bile reflux index (BRI), Helicobacter pylori status, clinical group, and chronic and acute inflammation at the cardia

\begin{tabular}{|c|c|c|c|c|c|}
\hline Variable & Total No & $\begin{array}{l}\% \text { with incomplete } \\
\text { IM }\end{array}$ & $\begin{array}{l}\text { Unadjusted odds } \\
\text { ratio }\end{array}$ & $95 \% \mathrm{Cl}$ & p Value* \\
\hline \multicolumn{6}{|l|}{ Sex } \\
\hline Female & 32 & 68.8 & 1.00 & & \\
\hline Male & 34 & 47.1 & 0.40 & $0.15-1.11$ & 0.087 \\
\hline \multicolumn{6}{|l|}{ BRI } \\
\hline$\leqslant 14$ & 45 & 51.1 & 1.00 & & \\
\hline$>14$ & 21 & 71.4 & 2.39 & $0.79-7.28$ & 0.18 \\
\hline \multicolumn{6}{|l|}{ H pylori } \\
\hline Negative & 47 & 63.8 & 1.00 & & \\
\hline Positive & 19 & 42.1 & 0.41 & $0.14-1.22$ & 0.17 \\
\hline \multicolumn{6}{|l|}{ Group } \\
\hline GORD & 42 & 57.1 & 1.00 & & \\
\hline NUD & 24 & 58.3 & 1.05 & $0.38-2.90$ & 1.00 \\
\hline \multicolumn{6}{|l|}{ Cardia $\mathrm{Cl}$} \\
\hline No & 22 & 63.6 & 1.00 & & \\
\hline Yes & 44 & 54.5 & 0.69 & $0.24-1.96$ & 0.60 \\
\hline \multicolumn{6}{|l|}{ Cardia Al } \\
\hline No & 44 & 65.9 & 1.00 & & \\
\hline Yes & 22 & 40.9 & 0.36 & $0.12-1.03$ & 0.067 \\
\hline $\begin{array}{l}\text { *Fisher's exc } \\
\text { GORD, gast } \\
\text { inflammation }\end{array}$ & ophageal & lux disease; NUD, & -ulcer dyspepsia; C & I, chronic & on; $\mathrm{Al}$, acute \\
\hline
\end{tabular}

(complete (type I) or incomplete (types II and III)) was evaluated (table 3$)$. There was a trend ( $>20 \%$ difference) for incomplete metaplasia to be associated with a BRI $>14, H$ pylori negative status, absence of acute inflammation at the cardia, and female sex. However, these factors did not reach formal statistical significance and the numbers of cases are small, making the data difficult to interpret (table 3).

Antral IM was found in 26 of 66 patients with cardia IM $(39 \%)$ but there were an additional 16 patients with isolated antral IM. While the score for antral IM contributed to BRI, its presence did not predict high values. Equal numbers of patients with metaplasia in the stomach (that is, 21/42) had a BRI above and below 14

\section{DISCUSSION}

The most striking finding in this study was that a histological index of bile reflux into the stomach was significantly associated with the presence of IM at the cardia. This is in keeping with a previous study in which we found that patients with Barrett's oesophagus (that is, IM in the oesophagus) had significantly higher BRI values than those with uncomplicated reflux oesophagitis or simple NUD. ${ }^{14}$ Interestingly, we found no statistically independent association between cardia IM and symptoms of GORD or with H pylori infection. While it is evident that a lack of statistical significance does not equate to absence of effect, our finding that increasing age, male sex, and chronic inflammation, but not $H$ pylori infection, were factors most closely associated with cardia IM is clearly in keeping with the known epidemiology of cardia cancer. The additional observation that a high BRI is also a significant risk factor for cardia IM gives important new information which may have a bearing on pathogenesis.

We identified chronic inflammation at the cardia in 106 of $226(47 \%)$ patients yet "carditis" has been claimed to be a universal finding whenever cardia-type mucosa is present and is indicative of GORD. ${ }^{15}{ }^{16}$ While it is conceded that small numbers of lymphocytes and plasma cells are frequently, if not universally, present in cardia mucosa, this does not necessarily represent "chronic inflammation". The mere presence of mononuclear cells cannot be equated with inflammation as otherwise the entire mucosa of the intestinal tract would be in a state of inflammation. It seems to us that some degree of lymphocytic infiltration, especially close to the squamocolumnar junction, is likely to be "physiological". As Weinstein puts $\mathrm{it}^{17}$ "these changes (carditis) could represent the consequence of gastric contents lapping at the shores of the oesophagus in health" (italics added). If it is acknowledged that the cardia has a "normal" complement of mononuclear cells, then the diagnosis of chronic inflammation requires a higher threshold, and this has been our approach. Some authors avoid the difficulties in diagnosing chronic inflammation at this site by defining "carditis" as infiltration by polymorphs. ${ }^{18}$ Using this criterion, equivalent to active chronic inflammation, we found "carditis" in only $45 / 226$ cases (19.9\%). Interestingly this is very close to the $18.4 \%$ prevalence of polymorph infiltration found by Der and colleagues ${ }^{16}$ yet these authors diagnosed chronic inflammation in all cases where cardia mucosa was present. In our series, acute and chronic inflammation at the cardia were significantly greater in $H$ pylori infected compared with non-infected patients but inflammation bore no significant association to clinical group. These findings run contrary to recent studies that emphasise the role of GORD ${ }^{39}$ but are entirely analogous to those reported by others. ${ }^{8}{ }^{19} 20$

With regard to the subtypes of IM found in cardia mucosa, we found a trend towards an association between incomplete IM and a high BRI, $H$ pylori negativity, absence of acute inflammation, and female sex but the groups were too small to yield significant associations. Previous work has examined IM subtypes at the cardia and their disease associations and found significant differences. For instance, Voutilainen and colleagues ${ }^{6}$ examined 235 patients with cardia IM and found that complete IM was associated with a pattern of gastritis characteristic of longstanding $H$ pylori infection while incomplete IM was associated with erosive oesophagitis, the lesion which predisposes to Barrett's oesophagus.

To our knowledge this is the first study that has demonstrated, albeit by indirect means, a link between bile reflux and cardia IM. This has potentially important implications for the development of cancer at the cardia. The connection between exposure to bile acids or their breakdown products and carcinogenesis at other sites is well known. The development of duodenal adenomas and carcinomas in patients with familial adenomatous polyposis and the predilection for adenocarcinoma to arise at the ampulla of Vater are thought to be bile related. ${ }^{21}$ The appearance of 
dysplasia and adenocarcinoma in gastric remnants following partial gastrectomy, and the development of carcinoma in the stomachs of experimental animals subjected to enterogastric reflux, have also been attributed to exposure to bile and/or duodenopancreatic juice. ${ }^{22}$ Finally, there is evidence that faecal bile acids in conjunction with dehydrogenating bacteria are implicated in the development of sporadic colorectal cancer. ${ }^{23}$ Thus bile acids have the potential not only to bring about IM at the cardia but also to generate the carcinogens which act on this altered mucosa to produce neoplasia. ${ }^{24}$ Changes in $\mathrm{pH}$ and the composition of the refluxate after regurgitation through the stomach could alter its carcinogenic potential ${ }^{25}$ and render it more damaging to the oesophagus. ${ }^{26}{ }^{27}$ Given the intrinsic instability of junctional sites and their predisposition to neoplasia, ${ }^{28}$ it is certainly plausible that exposure of the cardia to duodenogastric reflux could contribute to the development of both IM and adenocarcinoma at this site. Nevertheless, our conclusions must remain speculative until confirmed by direct measurements of intragastric bile content.

\section{ACKNOWLEDGEMENTS}

We are grateful to Knoll Ltd for an unrestricted grant in support of Dr Neville during the conduct of this study. Thanks are due to Stephen Ryan and Peter Jackson for technical assistance, Tim Branch for pathology computer searches, and to Les Beck for slide retrieval. Paul Moayyedi is funded by a UK MRC Health Services Research Training Fellowship.

\section{Authors' affiliations}

M F Dixon, N P Mapstone, Academic Unit of Pathology, University of Leeds, UK

P M Neville, P Moayyedi, A T R Axon, Centre for Digestive Diseases, General Infirmary at Leeds, Leeds, UK

\section{REFERENCES}

1 Owen DA. Stomach. In: Sternberg SS ed. Histology for pathologists, 2nd edn. Philadelphia: Raven Lippincott, 1997:481.

2 Fenoglio-Preiser CM, Noffsinger AE, Stemmerman GN, et al. Gastrointestinal pathology. An atlas and text, 2nd edn. Philadelphia: Raven Lippincott, 1999:133.

3 Chandrasoma PT, Lokuhetty DM, Demeester TR, et al. Definition of histopathologic changes in gastroesophageal reflux disease. Am J Surg Pathol 2000;24:344-51.

4 Carton E, Caldwell MTP, McDonald G, et al. Specialized intestinal metaplasia in patients with gastro-oesophageal reflux disease. Br J Surg 2000;87: 116-21.

5 Spechler SJ, Zeroogian JM, Antonioli DA, et al. Prevalence of metaplasia at the gastro-oesophageal junction. Lancet 1994;344:1533-6.
6 Voutilainen $M$, Farkkila $M$, Juhola $M$, et al. Complete and incomplete intestinal metaplasia at the oesophagogastric junction: prevalences and associations with endoscopic erosive oesophagitis and gastritis. Gut 1999;45:644-8

7 Ruol A, Parenti A, Zaninotto G, et al. Intestinal metaplasia is the probable precursor of adenocarcinoma in Barrett oesophagus and adenocarcinoma of the gastric cardia. Cancer 2000;88:2520-8.

8 Goldblum JR, Vicari JJ, Falk GW, et al. Inflammation and intestinal metaplasia of the gastric cardia: The role of gastroesophageal reflux and H. pylori infection. Gastroenterology 1998;114:633-9.

9 Oberg S, Peters JH, Demeester TR, et al. Inflammation and specialized intestinal metaplasia of cardiac mucosa is a manifestation of gastroesophageal reflux disease. Ann Surg 1997;226:522-30.

10 Marshall EEK, Anggiansah A, Owen WJ. Bile in the oesophagus: clinical relevance and ambulatory detection. Br J Surg 1997;84:21-8.

11 Sobala GM, O'Connor HJ, Dewar EP, et al. Bile reflux and intestinal metaplasia in gastric mucosa. J Clin Pathol 1993;46:235-40.

12 Dixon MF, Genta RM, Yardley JH, et al. Classification and grading of gastritis: The updated Sydney system. Am J Surg Pathol 1996;20: $1161-81$.

13 Shah KA, Deacon AJ, Dunscombe P, et al. Intestinal metaplasia subtyping: evaluation of Gomori's aldehyde fuchsin for routine diagnostic use. Histopathology 1997:31:277-83.

14 Dixon MF, Neville PM, Mapstone NP, et al. Bile reflux gastritis and Barrett's oesophagus: further evidence of a role for duodenogastro-oesophageal reflux? Gut 2001;49:359-63.

15 Rotterdam H. Pathology of the gastric cardia. Verh Disch Ges Pathol 1999;83:37-42.

16 Der R, Tsao-Wei DD, Demeester T, et al. Carditis: A manifestation of gastroesophageal reflux disease. Am J Surg Pathol 2001;25:245-52.

17 Weinstein WM, Bogoch ER, Bowes KL. The normal human esophageal mucosa: a histological reappraisal. Gastoenterology 1975;68:40-4.

18 El-Zimaity HMT, Verghese VJ, Ramchatesingh J, et al. The gastric cardia in gastro-oesophageal disease. J Clin Pathol 2000;53:619-25.

19 Chen YY, Antonioli DA, Spechler SJ, et al. Gastroesophageal reflux disease versus Helicobacter pylori infection as the cause of gastric carditis. Mod Pathol 1998;1 1:950-6.

20 El-Serag HB, Sonnenberg A, Jamal MM, et al. Characteristics of intestinal metaplasia in the gastric cardia. Am $j$ Gastroenterol 1999:94:622-7.

21 Spigelman AD, Williams CB, Grenowska M, et al. Gastro-duodenal adenomas in polyposis-Is bile the missing link? Gut 1989;30:A71011.

22 Miwa K, Hattori T, Miyazaki I. Duodenogastric reflux and foregut carcinogenesis. Cancer 1995;75:1426-32.

23 Nagengast FM. Bile acids and colon carcinogenesis. Scand J Gastroenterol 1988;23(suppl 154):76-81

24 Busby WF, Shuker DEG, Charnley G, et al. Carcinogenicity in rats of the nitrosated bile acid conjugates $\mathrm{N}$-nitrosoglycocholic acid and $\mathrm{N}$-nitrosotaurocholic acid. Cancer Res 1985;45:1367-71.

25 Hofmann AF, Mysels KJ. Bile acid solubility and precipitation in vitro and in vivo: the role of conjugation, $\mathrm{pH}$ and $\mathrm{Ca}^{2+}$ ions. J Lipid Res 1992;33:617-26.

26 Kaver WKH, Peters JH, DeMeester TR, et al. Mixed reflux of gastric and duodenal juices is more harmful to the esophagus than gastric juice alone. The need for surgical therapy re-emphasized. Ann Surg 1995;222:525-33.

27 Nehra D, Howell P, Williams CP, et al. Toxic bile acids in gastro-oesophageal reflux disease: influence of gastric acidity. Gut gastro-oesophageal

28 van Zanten SJOV, Dixon MF, Lee A. The gastric transitional zones: Neglected links between gastroduodenal pathology and Helicobacter ecology. Gastroenterology 1999;116:1217-29. 